\title{
Marginally low mass ratio close binary system V1191 Cyg
}

\author{
B. Ulaş ${ }^{\text {a,* }}$, B. Kalomeni ${ }^{\text {a,b }}$, V. Keskin ${ }^{\text {a }}$, O. Köse ${ }^{\text {a }}$, K. Yakut ${ }^{\text {a }}$ \\ ${ }^{a}$ Department of Astronomy and Space Sciences, University of Ege, 35100 İzmir, Turkey \\ ${ }^{\mathrm{b}}$ Department of Physics, Izmir Institute of Technology, 35430 Izmir, Turkey
}

\section{A R T I C L E I N F O}

\section{Article history:}

Received 10 May 2011

Received in revised form 26 May 2011

Accepted 2 June 2011

Available online 25 June 2011

Communicated by P.S. Conti

\section{Keywords:}

Stars: binaries: eclipsing

Stars: binaries: close

Stars: individual: V1191 Cyg

Stars: fundamental parameters

\begin{abstract}
A B S T R A C T
In this study, we present photometric and spectroscopic variations of the extremely small mass ratio ( $q \simeq 0.1$ ) late-type contact binary system V1191 Cyg. The parameters for the hot and cooler companions have been determined as $M_{\mathrm{h}}=0.13(1) M_{\odot}, M_{\mathrm{c}}=1.29(8) M_{\odot}, R_{\mathrm{h}}=0.52(15) R_{\odot}, R_{\mathrm{c}}=1.31(18) R_{\odot}, L_{\mathrm{h}}=0.46$ (25) $L_{\odot}, L_{\mathrm{C}}=2.71(80) L_{\odot}$, the separation of the components is $a=2.20$ (8) $R_{\odot}$ and the distance of the system is estimated as 278 (31) pc. Analyses of the times of minima indicates a period increase of $\frac{d P}{d t}=1.3(1) \times 10^{-6}$ days $/ \mathrm{yr}$ that reveals a very high mass transfer rate of $\frac{d M}{d t}=2.0(4) \times 10^{-7} \mathrm{M}_{\odot} / \mathrm{yr}$ from the less massive component to the more massive one. New observations show that the depths of the minima of the light curve have been interchanged.
\end{abstract}

(c) 2011 Elsevier B.V. All rights reserved.

\section{Introduction}

Modelling the evolution of late-type contact binary systems (LTCBs) is highly complicated. Yakut and Eggleton (2005) (hereafter YE05) modelled late-type close binary systems by assuming conservative and non-conservative cases. The authors proposed that a detached configuration can evolve into a semi-detached and then to a contact configuration. The mass ratio of the components reverses through evolution. Therefore, currently observed low mass star could be initially the more massive one in a binary system. The mass ratio of the components, which is related to the angular momentum loss and mass transfer, is one of the crucial parameter in the evolution of close binary systems. Apart from V1191 Cyg, there exist rare well-known LTCBs with a small mass ratio (e.g. SX Crv, V870 Ara, FG Hya, $\epsilon$ CrA, CK Boo, FP Boo, DN Boo, AH Cnc; see YE05, Şenavcı et al., 2008; Yakut et al., 2009).

V1191 Cyg was classified as a W UMa type system by Mayer (1965). Many years later, the first photometric study of the system was made by Pribulla et al. (2005a) and they derived the geometric elements of the binary system without a spectroscopic study. The authors gave the mass ratio of the components as 0.09 and reported orbital period increase of $4.2 \times 10^{-6} \mathrm{yr}^{-1}$. Rucinski et al. (2008) studied the radial velocity curve of V1191 Cyg and determined the spectroscopic mass ratio, orbital period, radial velocity amplitudes, and the velocities of the center of mass of the system.

\footnotetext{
* Corresponding author. Fax: +90 2323731403.

E-mail address: bulash@gmail.com (B. Ulaş).
}

However, the authors also mentioned that the system is indeed a difficult spectroscopic target because of its faintness.

\section{New observations}

The observations carried out with the $40-\mathrm{cm}$ telescope at Ege University Observatory. This telescope equipped with the Apogee CCD camera. The light curve of the system and its times of minima light are obtained in the $B, V$, and $R$ bands in three nights in 2008 (Fig. 1a). Comparison and check stars were selected as GSC 31591409 and GSC 3159-1701, respectively. Because of the suspected case of GSC 3159-1701, we also chose an additional check star, GSC 3159-1593, to examine any variability of the comparison star. 237,274 and 280 points were obtained in $B, V$, and $R$ bands, respectively. The data are reduced by using IRAF (DIGIPHOT/APPHOT) packages. Standard deviations of observations are estimated for $B, V$, and $R$ bands as $0.002,0.001$, and 0.001 , respectively. In Table 1 we list all the observed data.

Due to stellar activity and/or inhomogeneous stellar surface, the light curve variation of the system shows maxima with different amplitudes (Fig. 1) known as O'Connell effect. We considered this effect in our light curve analysis and further discussed it in Section 3. The depths of the primary minima are $0^{m} .34,0^{m} .31$ and $0^{m} .30$ in $B, V$ and $R$ filters, respectively. The secondary minima are shallow by $0^{m} .060$ in $B, 0^{m} .055$ in $V$, and $0^{m} .040$ in $R$ band. Duration of the primary and secondary minima are $3^{h} .1$ and $3^{h} .2$, respectively. The comparison of light curves presented in this study with earlier ones shows that the depth and the shape of the light curve have been changed. We will discuss this in the last section. 

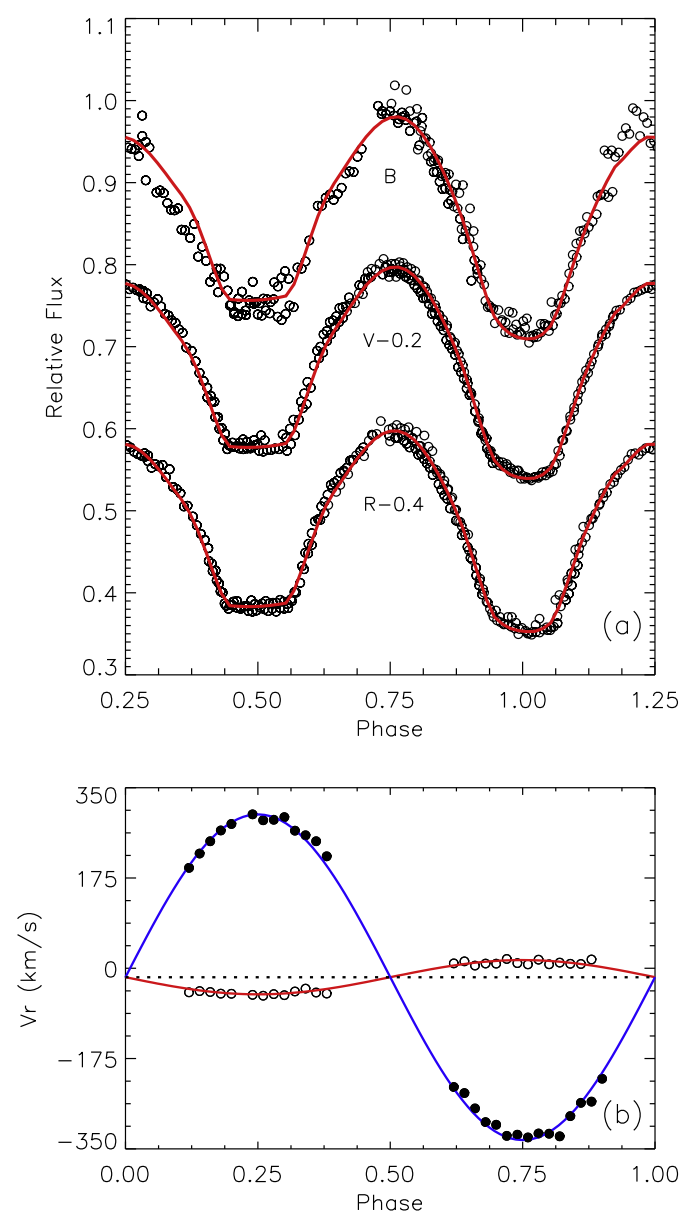

Fig. 1. (a) The observed and computed (solid line) light and (b) radial velocity curves of V1191 Cyg. Light curves in V and R bands are moved by a value of -0.20 and -0.40 , respectively, for a good visibility. Observed radial velocities are obtained from Rucinski et al. (2008). The continous lines are estimated from simultaneous solution.

We obtained four new minima times throughout these observations. They are given with those published in Table 2 with their errors. Using Table 2 we derived a new linear ephemeris (Eq. (1)) and used it for analyses.

$H J D M i n I=2454635.5231(7)+0.3133896(3) \times E$

\section{Eclipse timings and period study}

Orbital period study of the system was made previously by Pribulla et al. (2005a). The authors reported very fast period increase of $\Delta P / P=4.22 \times 10^{-6} \mathrm{yr}^{-1}$. Recent observations show that the deeper primary is the transit eclipse. Therefore, we define the transit minimum as a primary minimum (I) and the occultation as a secondary minimum (II). We present minima times obtained in this study together with those of earlier studies in Table 2 .

We solved the $O-C$ curve to find a parabolic variation that can be described as a mass transfer from the less massive companion to the more massive one. A total of 35 data obtained by photometric/CCD observations are used to study the period variation of the system. The weighted least-squares method is used in order to determine the parameters of the upward parabolic variation. The residuals $\left(\Delta T_{I}\right)$ indicate a quadratic solution (Fig. 2 ). In order to estimate the light elements given in Eq. (2) differential correction method is used. Using a weighted least squares solution we obtain the parameters as:
Table 1

Observational points of V1191 Cyg. Heliocentric Julian Date, phase, and magnitudes $(B, V$, and $R)$ are listed. Table 1 is given in its entirety in the electronic edition of this paper. A portion of it is shown here for guidance regarding its form and content.

\begin{tabular}{llll}
\hline $\begin{array}{l}\text { HJD } \\
2454600+\end{array}$ & Phase & $\begin{array}{l}\Delta \mathrm{m} \\
\mathrm{mag}\end{array}$ & Filter \\
\hline 30.3282 & & 0.047 & $\mathrm{~B}$ \\
30.3304 & 0.425 & 0.041 & $\mathrm{~B}$ \\
30.3325 & 0.430 & 0.080 & $\mathrm{~B}$ \\
30.3346 & 0.437 & 0.078 & $\mathrm{~B}$ \\
30.3367 & 0.444 & 0.088 & $\mathrm{~B}$ \\
30.3388 & 0.451 & 0.095 & $\mathrm{~B}$ \\
30.3410 & 0.458 & 0.086 & $\mathrm{~B}$ \\
30.3431 & 0.464 & 0.080 & $\mathrm{~B}$ \\
\hline
\end{tabular}

Table 2

The times of minima light of V1191 Cyg.

\begin{tabular}{llll}
\hline HJD* Min & Ref. & HJD Min & Ref. \\
\hline 49587.3879 & 1 & 52905.305 & 6 \\
49599.4536 & 1 & 52905.4591 & 6 \\
49608.384 & 1 & 53156.4815 & 6 \\
49619.3527 & 1 & 53512.4895 & 6 \\
49619.5106 & 1 & 53258.4871 & 7 \\
49621.3904 & 1 & 53340.2859 & 7 \\
49644.2651 & 2 & 53612.4622 & 8 \\
49644.4228 & 2 & 53685.642 & 9 \\
50672.455 & 3 & 53915.5113 & 10 \\
52413.445 & 4 & 53921.4649 & 10 \\
52445.4067 & 4 & 53934.4683 & 10 \\
52456.3748 & 4 & 54025.3548 & 11 \\
52465.4622 & 4 & 54049.6383 & 12 \\
52528.3033 & 4 & $54630.3527(3)$ & 13 \\
52548.3544 & 4 & $54635.3660(2)$ & 13 \\
52901.5459 & 5 & $54635.5230(5)$ & 13 \\
52902.3257 & 6 & $54636.4643(2)$ & 13 \\
52902.4848 & 6 & &
\end{tabular}

References for Table 2. 1 - Agerer and Hubscher (1995), 2 - Agerer and Hubscher (1996), 3 - Agerer and Hubscher (1998), 4 - Pribulla et al. (2002), 5 - Hubscher (2005), 6 - Pribulla et al. (2005b), 7 - Hubscher et al. (2005), 8 - Hubscher et al. (2006), 9 - Nelson (2006), 10 - Parimucha et al. (2007), 11 - Hubscher (2007), 12 Nelson (2007), 13 - present study.

$$
\begin{aligned}
\text { HJDMinI }= & 2452548.5135(1)+0.31338491(2) \times E \\
& +5.5(3) \times 10^{-10} \times E^{2} .
\end{aligned}
$$

\section{Simultaneous solutions of light and radial velocity curves}

The only photometric light curve study of the system was published by Pribulla et al. (2005a) who applied the RocHe code to the $B V R$ data of two nights taken at the Stara Lesna Observatory. Pribulla first performed a grid search to the data to evaluate the mass ratio and resulted the analysis with geometric parameters $i=80^{\circ} .4, q=0.094$, and $f=0.46$. In addition, Pribulla also mentioned about the possibility of the presence of a third body. The radial velocity of the system was studied by Rucinski et al. (2008). Spectroscopic orbital elements was given as $V_{0}=-16.82 \mathrm{~km} / \mathrm{s}$, $q=0.107, K_{1}=33.68 \mathrm{~km} / \mathrm{s}$, and $K_{2}=315.52 \mathrm{~km} / \mathrm{s}$ (ibid). Rucinski et al. (2008) reported smaller mass ratio for the system than an ordinary W-type W UMa system.

New light curves and radial velocity curves of Rucinski et al. (2008) simultaneously have been solved by using the program PHOEBE (Prša and Zwitter, 2005), which uses the WD code (Wilson and Devinney, 1971). In the solution we used weighted light curves which are constructed according to the standard deviation of each filter. The effective temperature of the cooler star is chosen accord- 

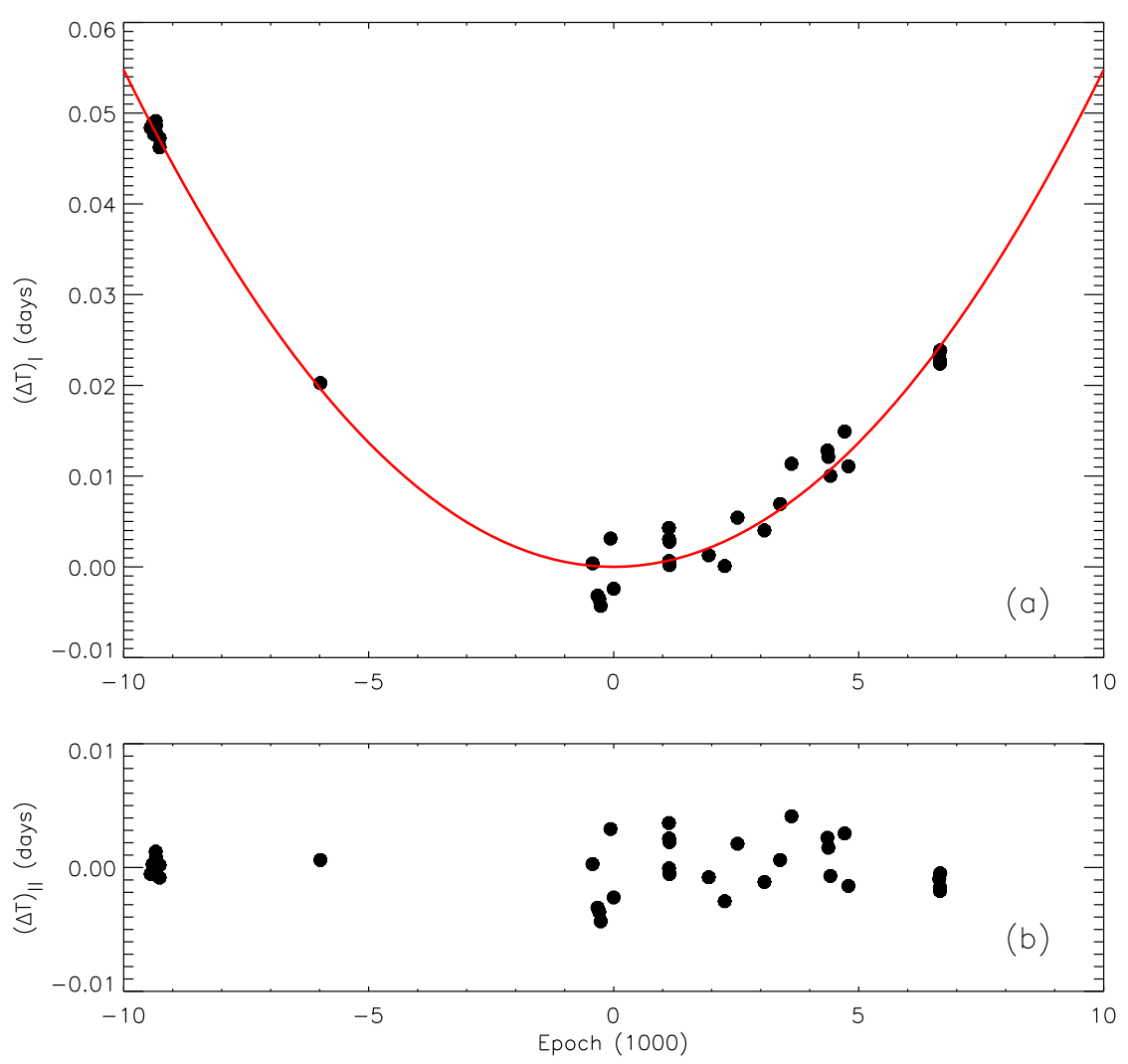

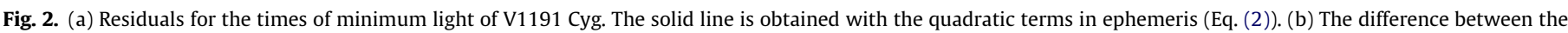
observations and the quadratic ephemeris.

ing to the spectroscopic study of Rucinski et al. (2008) in which its spectral type was given as F6V. The albedos, $A_{\mathrm{h}}$ and $A_{\mathrm{c}}$ are adopted from Rucinski (1969), and the values of the gravity darkening coefficients, $g_{\mathrm{h}}$ and $g_{\mathrm{c}}$, are taken from Lucy (1967). The logarithmic limb-darkening law are used with coefficients adopted from van Hamme (1993) for a solar composition star (Table 3). The adjustable photometric parameters are orbital inclination, $i$, surface potential, $\Omega_{h}=\Omega_{c}=\Omega$, temperature of the hot component, $T_{\mathrm{h}}$, luminosity, $L_{\mathrm{h}}$, mass ratio, $q$, the velocity of the center of mass, $V_{0}$, semi-major axis, $a$, the time of minima, $T_{0}$, and the orbital period, $P$ are set as free parameters.

The light curve shows asymmetry between two maxima probably because of the presence of the stellar spots. We assumed a cool active region on the surface of the cooler component and represented it with a spot to adjust the difference between two maxima. The code does not give results for simultaneous solutions where the spot parameters are treated as free parameters. In order to obtain the best parameters, we tested different solutions by changing the spot parameters. The solution with the smallest standard deviation is regarded as the best solution for spot parameters. Co-latitude $(\beta)$, longitude $(\lambda)$, fractional radius $(r)$, and the temperature factor $(t)$ of the spot are given in Table 3. Spot parameters obtained from the LC solutions do not represent a unique spot but the total active area on the stellar surface. Table 3 also summarizes results of the analysis. $B, V$, and $R$ light curves and velocity curves that are computed using the determined parameters are shown with solid lines in Fig. 1a. The solution presented in this study is the first simultaneous light and radial velocity data solution of the system.

\section{Results and conclusion}

$B, V$, and $R$ light curves of the system have been solved simultaneously with the spectroscopic study of Rucinski et al. (2008). We derived the orbital and the physical parameters of the components. Using obtained parameters we could estimate the filling factor $\left(f=\frac{\Omega_{\text {in }}-\Omega}{\Omega_{\text {in }}-\Omega_{\text {out }}}\right)$ from the inner $\left(\Omega_{\text {in }}\right)$ to the outer critical surface $\left(\Omega_{\text {out }}\right)$ as 0.74 . The distance of the system to the Sun estimated as $278 \mathrm{pc}$ by using observed parameters (Table 4 ). This is the first distance value given for V1191 Cyg. During the calculations, effective temperature and absolute magnitude of the Sun are taken as $5780 \mathrm{~K}$ and $4.75 \mathrm{mag}$, respectively. We compared our result with the well-known contact binaries using YE05 tables and figures. The physical parameters obtained from our analysis of V1191 Cyg seem to be in a good agreement with the well-known LTCBs. Like the other W-subtype secondaries, the less massive component of V1191 Cyg is overluminous and oversized in YE05 diagrams. While the secondary component of the system appears to be below the ZAMS, the massive and the cooler component is situated close to the TAMS. This phenomenon was discussed in detail by YE05, Kalomeni et al. (2007), Stepien (2006), and Gazeas and Niarchos (2006).

Light variations at maxima and minima depths have been detected for the first time. The light curves of Pribulla et al. (2005a) indicate that the occultation minimum is deeper than the transit by $\sim 0.01 \mathrm{mag}$ in $\mathrm{R}$ band. However, light curves presented in this study (Fig. 1(a)) show that the deeper minimum is the transit and the shallower one is the occultation. The occultation minimum is shallower by $\sim 0.04$ mag in $\mathrm{R}$ band.

There are two subgroups of A- and W- types for LTCBs according to Binnendijk (1970) classification. If the more massive component (the hotter one) is eclipsed during the deeper minimum, then we are dealing with an A-type system. If the less massive star is the hotter one, the system is W-type. FG Hya (Qian and Yang, 2005) and EM Psc (Qian et al., 2008) show an interchange between this two subgroups which is consistent with the model of YE05. The light curves of Pribulla et al. (2005a) show that V1191 Cyg is a W-type system whereas our observations show that the minima 
Table 3

The light and velocity curve results and their formal $1 \sigma$ errors for V1191 Cyg. The indices $h$ and $c$ refer to the hot and cooler components, respectively. See text for details.

\begin{tabular}{ll}
\hline Parameter & Value \\
\hline$i\left({ }^{\circ}\right)$ & $80.1(5)$ \\
$q=M_{\mathrm{h}} / M_{\mathrm{c}}$ & $0.105(2)$ \\
$a\left(\mathrm{R}_{\odot}\right)$ & $2.192(72)$ \\
$V_{0}\left(\mathrm{kms}^{-1}\right)$ & $-17.3(3)$ \\
$\Omega_{h}=\Omega_{c}$ & $1.924(194)$ \\
$T_{\mathrm{h}}(\mathrm{K})$ & $6610(200)$ \\
$T_{\mathrm{c}}(\mathrm{K})$ & 6500 \\
Fractional radius of hot comp. & $0.237(79)$ \\
Fractional radius of cooler comp. & $0.598(70)$ \\
$A_{\mathrm{h}}=A_{\mathrm{c}}$ & 0.6 \\
$g_{\mathrm{h}}=g_{\mathrm{c}}$ & 0.32 \\
Luminosity ratio: $: L_{c}$ & \\
$B$ & $0.880(50)$ \\
$V$ & $0.878(39)$ \\
$R$ & $0.876(20)$ \\
Spot parameters & \\
$\beta\left({ }^{\circ}\right)$ & 30 \\
$\lambda\left(^{\circ}\right)$ & 290 \\
$r$ & 20 \\
$t$ & 0.9 \\
\end{tabular}

Table 4

Absolute parameters of V1191 Cyg. The standard errors $1 \sigma$ in the last digit are given in parentheses. $\mathrm{H}$ and $\mathrm{C}$ stand for the hot and the cooler components, respectively.

\begin{tabular}{llll}
\hline Parameter & Unit & $\mathrm{H}$ & $\mathrm{C}$ \\
\hline Mass (M) & $\mathrm{M}_{\odot}$ & $0.13(1)$ & $1.29(8)$ \\
Radius (R) & $\mathrm{R}_{\odot}$ & $0.52(15)$ & $1.31(18)$ \\
Temperature $\left(\mathrm{T}_{\text {eff }}\right)$ & $\mathrm{K}$ & $6610(200)$ & 6500 \\
Luminosity (L) & $\mathrm{L}_{\odot}$ & $0.46(8)$ & $2.71(44)$ \\
Surface gravity (logg) & $\mathrm{cms}^{-2}$ & 4.12 & 4.31 \\
Absolute magnitude $\left(\mathrm{M}_{V}\right)$ & $\mathrm{mag}$ & 5.73 & 3.82 \\
Period change rate $(\dot{P})$ & $\mathrm{d} / \mathrm{yr}$ & $1.3(1) \times 10^{-6}$ & \\
Mass transfer ratio $(\dot{M})$ & $\mathrm{M}_{\odot} / \mathrm{yr}$ & $2.0(4) \times 10^{-7}$ & \\
Distance $(\mathrm{d})$ & $\mathrm{pc}$ & $278(31)$ & \\
& & &
\end{tabular}

of the light curve have been interchanged. Apart from the long period evolution (e.g. heat transfer between components) of LTCB systems, short period variation (e.g. efficiently stellar activity) can affect this phenomena. V1191 Cyg shows similar characteristic properties of FG Hya and EM Psc.

V1191 Cyg with binaries having small mass ratio and short orbital period form a small but important sub-group of binary zoo.
Short period and small mass ratio in contact binaries play a crucial role to explain astrophysical phenomena that are not well understood yet (i.e. merge of close/contact binaries, blue stragglers and the formation of FK Com stars) (YE05; Arbutina, 2009; Jiang et al., 2010). It is generally believed that these kinds of systems can be the progenitors of the FK Com systems and blue stragglers.

\section{Acknowledgments}

This study was supported by the Turkish Scientific and Research Council (TÜBITAK 109T047) and Ege University Research Fund. K.Y. and V.K. acknowledges support by the Turkish Academy of Sciences (TÜBA). The authors thank to E.R. Pekünlü for his valuable comments.

\section{References}

Agerer, F., Hubscher, J., 1995. IBVS, 4222, 1.

Agerer, F., Hubscher, J., 1996. IBVS, 4383, 1.

Agerer, F., Huebscher, J., 1998. IBVS, 4606, 1.

Arbutina, B., 2009. MNRAS 394, 501.

Binnendijk, L., 1970. VA 12, 217.

Gazeas, K.D., Niarchos, P.G., 2006. MNRAS 370, L29.

Hubscher, J., 2005. IBVS, 5643, 1.

Hubscher, J., Paschke, A., Walter, F., 2005. IBVS, 5657, 1.

Hubscher, J., Paschke, A., Walter, F., 2006. IBVS, 5731, 1.

Hubscher, J., 2007. IBVS, 5802, 1.

Jiang, D., Han, Z., Wang, J., Jiang, T., Li, L., 2010. MNRAS 405, 2485.

Kalomeni, B., Yakut, K., Keskin, V., Değirmenci, Ö.L., Ulaş, B., Köse, O., 2007. AJ 134, 642.

Lucy, L.B., 1967. Zeit. Astrophys. 65, 89.

Mayer, P., 1965. Bulletin of the Astronomical Institutes of Czechoslovakia 16, 255.

Nelson, R.H., 2006. IBVS, 5672, 1.

Nelson, R.H., 2007. IBVS, 5760, 1.

Parimucha, S., et al., 2007. IBVS, 5777, 1.

Pribulla, T., Vanko, M., Parimucha, S., Chochol, D., 2002. IBVS, 5341, 1.

Pribulla, T., Vaňko, M., Chochol, D., Parimucha, Š., Baludanský, D., 2005a. Ap\&SS 296, 281.

Pribulla, T., et al., 2005b. IBVS, 5668, 1.

Prša, A., Zwitter, T., 2005. ApJ 628, 426.

Qian, S., Yang, Y., 2005. MNRAS. 356, 765.

Qian, S.-B., He, J.-J., Soonthornthum, B., Liu, L., Zhu, L.-Y., Li, L.-J., Liao, W.P., Dai, Z.-B., 2008. AJ 136, 1940.

Rucinski, S.M., 1969. Acta Astron. 19, 245.

Rucinski, S.M. et al., 2008. AJ 136, 586

Senavcı, H.V., Nelson, R.H., Özavcı, I., Selam, S.O., Albayrak, B., 2008. NewA 13, 468.

Stepien, K., 2006. ActaA 56, 199.

van Hamme, W., 1993. AJ 106, 2096.

Wilson, R.E., Devinney, E.J., 1971. ApJ 166, 605.

Yakut, K., Eggleton, P.P., 2005. ApJ 629, 1055

Yakut, K. et al., 2009. A\&A 503, 165. 